\title{
Materials Genome Initiative: Advances and Initiatives
}

\author{
Meredith Drosback
}

When President Barack Obama announced the Materials Genome Initiative (MGI) in June 2011, it brought renewed attention to the critical role that advanced materials play in everyday life. From electronics for the next generation of cell phones, to more fuel-efficient, lightweight automobiles, to lifesaving medical implants, materials are fundamental to most technology breakthroughs. Remaining globally competitive in the $21^{\text {st }}$ century will require increasingly rapid technological innovation, and the 20-year timeframe currently required to get a new material to market is simply too long. To address this, the aim of MGI is straightforward: cut in half the time and cost to discover, develop, and deploy these high-tech materials in the U.S. marketplace.

The Materials Genome Initiative deliberately focuses on this concept of time. It cuts across the entire materials development continuum from discovery at the bench to deployment for practical use, and aims to accelerate each step in the process. The Materials Genome Initiative is as much about advancing fundamental research for discovery as it is about improved systems design, innovations in processing and manufacturing, and understanding raw material lifecycles.

\section{The Components of MGI}

The Materials Genome Initiative has two core objectives: developing a materials innovation infrastructure and incentivizing a change in culture. The white paper introducing MGI, The Materials Genome Initiative for Global Competitiveness, available at www.whitehouse.gov /mgi, captured the vision for this infrastructure illustrated in Figure 1.

Three basic elements of modern science-computational, experimental, and data tools - are presented on the interior of Figure 1. Within the context of MGI, data is sourced both from experiment and

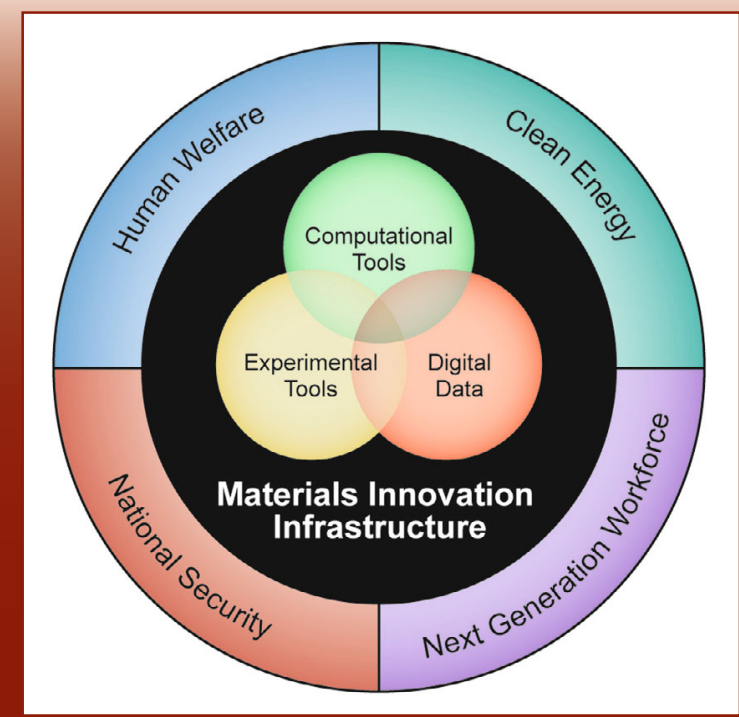

Figure 1. An overview of the MGI, showing the key components of the infrastructure and areas of application. (Credit: Materials Genome Initiative for Global Competitiveness, NSTC, Washington, D.C. 2011; www. whitehouse. gov/sites/default/files/ microsites/ostp/materials genome_initiative-final. pdf.) 


\section{Recent Progress}

What began as a modest $\$ 63$ million federal investment in fiscal year (FY) 2012 has grown to include five federal agencies: Department of Defense (DOD), Department of Energy (DOE), NASA, National Institute of Standards and Technology (NIST), and National Science Foundation (NSF).

Stakeholders from the private sector have also embraced the challenge, investing hundreds of millions of dollars on their own in support of MGI and its goals.

For example, two of the new National Network for Manufacturing Innovation (NNMI) institutes, announced in May 2013, are directly related to MGI principles. The DOD will lead the Lightweight and Modern Metals Manufacturing Innovation Institute and DOE is investing in a Clean Energy Manufacturing Innovation Institute on wide bandgap semiconductor power electronic devices. Both institutes will become part of a broader network of regional hubs to accelerate development and adoption of advanced manufacturing technologies. (Additional information on NNMI and its institutes can be accessed at www.manufacturing.gov /nnmi.html.)

NIST announced its intent in June 2013 to launch a Center of Excellence in Advanced Materials and awarded the center in December to a team led by Northwestern University, the University of Chicago, and Argonne National Laboratory, with partners QuesTek Innovations, ASM International, and Fayetteville State University. Capitalizing on the breadth of expertise within this public-private partnership and access to NIST's capabilities, the center will develop the next generation of computational tools, databases, and experimental techniques.

As the NIST center demonstrates, MGI is not only a federal initiative, In fact, participation and widespread adoption by the community is crucial to its long-term success. When MGI celebrated its two-year anniversary in June 2013, companies, universities, and scientific societies demonstrated their support with more than 20 new commitments to mark that milestone. (Access a fact sheet on these commitments at www. whitehouse .gov/sites/default/files/microsites lostp/2013_mgi_annoucements.pdf.)

Community interest in MGI has also catalyzed new efforts in regional collaboration. Five universities committed to host regional workshops on MGI for academic and industrial stakeholders to amplify local efforts in materials science and engineering innovation and highlight new opportunities for collaboration. Northeastern University hosted the first workshop in early October 2013. Workshops hosted by the University of Southern California, Rice University, Georgia Institute of Technology, and University of Illinois will follow throughout the spring of 2014.

\section{Strategic Plan}

One of the major federal activities of 2013 has been the development of the first strategic plan for MGI, due for release in the spring of 2014. The plan will focus on four goals. First, MGI will enable a paradigm shift in culture by encouraging and supporting integrated and collaborative research and development work across materials science and engineering. The second goal is to integrate experiment, computation, and theory. This goal includes not only developing new tools and techniques, but also linking these together in an integrated, iterative fashion.

MGI must also begin to build the foundation for a materials data infrastructure. Enabling the storage, curation, and reuse of data for a wide array of users will be a critical step for MGI's success, yet many questions still need to be answered about the design and implementation of the supporting infrastructure. Finally, it will be crucial to equip the next-generation workforce with the background and experience to work productively in collaborative teams.

In addition to presenting the MGI's goals and near-term steps toward meeting them, the strategic plan will highlight selected science and technology grand challenges, using input that has come from the community. NIST and DOE cohosted two workshops in 2013 to convene leaders from academia and industry, asking these participants to identify the major scientific and technical roadblocks in nine materials classes or applications. The June workshop explored challenges faced by several classes of hard materials, with breakout sessions on lightweight and structural materials, catalysts, correlated materials, electronics and photonics, and energy storage. November's event focused on soft materials, including polymers, polymer composites, biomaterials, and organic electronics. These workshops have proven useful, both for the community in identifying and exploring common challenges, and for the federal agencies that will have the opportunity to consider this input as they develop future MGI programs.

\section{Outlook}

The future that MGI envisions is one in which scientists and engineers work together seamlessly, putting new discoveries to use in solving some of the U.S.'s greatest challenges. While work remains to be done to make this vision a reality, support for and interest in the MGI continues to grow. Beyond the investment of federal agencies, it is the broader community of scientists, engineers, designers, and manufacturers whose participation in and implementation of MGI ultimately will meet the challenge.

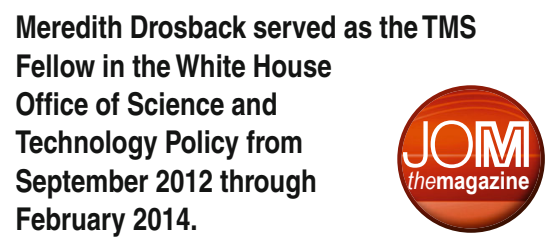

